\title{
Irritable Bowel Syndrome and Quality of Life in Women With Fecal Incontinence
}

\author{
Alayne D. Markland, DO, MSc, * J. Eric Jelovsek, MD, † David D. Rahn, MD, + Lu Wang, PhD, $\S$ \\ Leah Merrin, BS, II Ashok Tuteja, MD, 9 Holly E. Richter, MD, PhD,** \\ Susan Meikle, MD, †† and On Behalf of the Pelvic Floor Disorders Network
}

\begin{abstract}
Objectives: The objectives of this work were to determine the prevalence of irritable bowel syndrome (IBS) and IBS subtypes in women presenting for fecal incontinence (FI) treatment and to assess the impact of IBS on FI symptoms and quality of life (QOL).

Methods: In this multicenter prospective cohort study, women reported at least monthly solid, liquid, or mucus FI. Rome III clinical criteria defined IBS. Women also self-reported having an IBS diagnosis. Baseline questionnaires included the following: Modified Manchester Health Questionnaire, Fecal Incontinence Severity Index, Bristol Stool Scale, Pelvic Floor Distress Inventory, and the Pelvic Floor Impact Questionnaire.

Results: Of the 133 women enrolled, 119 completed Rome III IBS questionnaires, and 111 reported on whether they had a previous diagnosis of IBS. The prevalence of IBS was $31 \%(95 \%$ confidence interval [CI], $22.9 \%-40.2 \%$ ) according to the Rome III IBS criteria. The most common subtypes were IBS-mixed (41\%) and IBS-diarrhea (35\%). Twenty-four (22\%) of 111 patients had a previous diagnosis of IBS. Among women who met Rome III IBS criteria, 23 (66\%) of 35 women had never had a diagnosis of IBS. Women with FI and IBS reported significantly worse QOL compared to women without IBS despite similar FI severity and stool consistency.
\end{abstract}

Conclusions: Irritable bowel syndrome negatively affects QOL and affects one third of women with FI presenting for care in tertiary centers. Our findings suggest that assessment of IBS symptoms and diagnosis may be important for women presenting for FI treatment.

Key Words: irritable bowel syndrome, fecal incontinence, accidental bowel leakage, women, functional bowel disorders, pelvic floor disorders, quality of life

(Female Pelvic Med Reconstr Surg 2016;00: 00-00)

E ecal incontinence (FI) affects $4 \%$ to $24 \%$ of women in the com- munity. ${ }^{1}$ Irritable bowel syndrome (IBS) is more common among women than men, and the worldwide prevalence varies

From the *Birmingham Veterans Affairs Medical Center, Department of Medicine, University of Alabama at Birmingham, Birmingham, AL; $\dagger$ Obstetrics, Gynecology, and Women's Health, Cleveland Clinic, Cleveland, OH; †Obstetrics and Gynecology, University of Texas Southwestern Medical Center, Dallas, TX; §Biostatistics, University of Michigan, Ann Arbor, MI, United States; IIObstetrics and Gynecology, University of California, San Diego, San Diego, CA; $\uparrow$ Medicine, Salt Lake City Veterans Affairs Medical Center, University of Utah, Salt Lake City, UT; **Obstetrics and Gynecology, University of Alabama at Birmingham, Birmingham, AL; and † Eunice Kennedy Shriver NICHD, National Institutes of Health, Bethesda, MD.

Reprints: Alayne Markland, DO, MSc, Department of Veteran Affairs, Geriatric Research, Education, and Clinical Center, University of Alabama at Birmingham Department of Medicine, Division of Gerontology, Geriatrics, and Palliative Care, BVAMC, GRECC 11-G, Room 8220, 700 S 19th St, Birmingham, AL 35233. E-mail: amarkland@aging.uab.edu.

The authors have declared they have no conflicts of interest.

Supported by grants from the Eunice Kennedy Shriver National Institute of

Child Health and Human Development (U10 HD054215, U10 HD041261,

U10 HD054214, U10 HD041267, U10 HD069025, U10 HD069010, U10

HD069006, U01 HD069013, U01 HD069031) and the National Institutes

of Health Office of Research on Women's Health.

ClinicalTrials.gov Identifier: NCT 00729144.

Copyright $\odot 2016$ Wolters Kluwer Health, Inc. All rights reserved.

DOI: $10.1097 /$ SPV.0000000000000358 considerably based on regional differences. ${ }^{2-4}$ In the absence of detectable organic causes, FI and IBS are considered functional gastrointestinal disorders and have a negative impact on quality of life (QOL). ${ }^{5,6}$ Irritable bowel syndrome is diagnosed by chronically recurring abdominal pain or discomfort along with altered bowel habits. ${ }^{7,8}$ Irritable bowel syndrome has 4 subtypes: IBS-C (constipation), IBS-D (diarrhea), IBS-M (mixed, equal diarrhea and constipation types), and IBS-U (unclassified). The Rome Foundation has developed diagnostic questionnaires in coordination with the Rome III committees to assist in diagnosing functional gastrointestinal disorders, including IBS. ${ }^{9}$ Previous studies using Rome criteria to diagnosis IBS reported that the prevalence of IBS in women with FI ranges from $16 \%$ to $45 \%{ }^{4,8}$ From other studies, $45 \%$ to $57 \%$ of women with IBS may have FI. ${ }^{10-13}$ Rates of IBS are also high among women with urinary incontinence $(18 \%)$ and pelvic organ prolapse $(18 \%))^{14}$

Risk factors for FI include age, diarrhea (stool consistency), sensation of urgency to defecate, obstetrical injury, obesity, prior surgery to the rectal area, and neurological diseases, such as stroke and multiple sclerosis. ${ }^{1}$ Risk factors for IBS are not well characterized but may include female sex, prior gastrointestinal infections, and psychological stressors. ${ }^{7}$ Less is known about the impact of IBS on the severity of FI and health-related QOL in women presenting for treatment for FI. ${ }^{15,16}$ Our objectives were to define the prevalence of IBS and IBS subtypes and to determine the impact of IBS on FI-related symptoms, including QOL, among women with FI who are presenting for care at tertiary medical centers.

\section{MATERIALS AND METHODS}

This was an ancillary analysis from a prospective multicenter cohort study designed to evaluate adaptive behaviors among women with bowel incontinence, the Adaptive Behaviors among women with Bowel Incontinence (ABBI) study. The ABBI study was conducted through the Pelvic Floor Disorders Network, a clinical trial network that consisted of 7 clinical sites at the time of this study and a data coordinating center. As part of a larger study evaluating adaptive behaviors among women with pelvic floor disorders, women with FI were recruited from referralbased specialty care clinics. In addition to evaluating adaptive behaviors, validated questionnaires on severity of symptoms and QOL were collected before treatment for FI (per usual care) and 3 months and 12 months after treatment. ${ }^{17}$ Each clinical site and the data coordinating center received institutional review board approval, and all participants provided written informed consent for research participation.

Women met eligibility criteria if they had FI consisting of liquid stool, solid stool, or mucus occurring at least monthly for 3 consecutive months and were planning to have treatment for FI. Women were excluded if they had prior rectal or colon cancer, inflammatory bowel disease, pelvic irradiation, a current or prior rectal fistula(e), removal of any portion of the colon/rectum, rectal prolapse, or severe neurological conditions. 
Participants completed validated questionnaires in person or by telephone before treatment. Validated questionnaires assessed IBS symptoms, impact on health-related QOL (HR-QOL) and health-related QOL, as well as other pelvic floor symptoms. Symptom-based diagnostic criteria, the Rome III criteria, assessed the presence or absence of IBS and was collected once, before treatment. These questionnaires are available online for use (http://www.romecriteria.org/questionnaires/). ${ }^{9}$ The Rome Foundation defined 2 diagnostic categories of IBS using the Rome III criteria. The first category was defined by meeting the clinical criteria for IBS with symptoms occurring at least monthly. The second category was defined as research criteria with symptoms occurring at least weekly. In this study, we categorized IBS according to the Rome III clinical criteria. In addition, participants self-identified themselves as having a prior diagnosis of IBS according to the question: "Have you ever been told by a medical professional that you have IBS?"

Participants completed several additional validated questionnaires relating to bowel symptoms including the Fecal Incontinence Severity Index (FISI) ${ }^{18}$ and the Colorectal Anal Distress Inventory. ${ }^{19}$ Condition-specific QOL questionnaires included the Modified Manchester Health Questionnaire (MMHQ) ${ }^{20}$ and Colorectal Anal Impact Questionnaire. ${ }^{19}$ The Medical Outcomes Survey SF-12 measured the impact of symptoms on healthrelated QOL. ${ }^{21}$ Urinary symptoms and symptoms related to pelvic organ prolapse were measured with the Urinary Distress Inventory and the Pelvic Organ Prolapse Distress Inventory, respectively. ${ }^{19}$

The MMHQ, a validated questionnaire on the overall impact of FI on condition-specific QOL, consisted of a total score and 8 domain scores (31 questions). ${ }^{20}$ Specific items were measured on a 5-point scale as 1, "never" to 5,"always." Total scores and domain scores both ranged from 0 to 100 . The FISI had four questions on incontinence of gas, mucus, liquid stool, and solid stool. ${ }^{18}$ The answer categories for frequency of incontinence symptoms were: 2 or more times a day, once a day, 2 or more times a week, once a week, 1 to 3 times per month, or never. Responses were weighted on a 1-to-20 severity scale, and a total FISI score was calculated (range, 0-61 for patient-weighted scores). For both measures, a higher score indicated a greater impact or greater severity of symptoms. A strong fecal urgency sensation was defined by a response as "often or always" on the MMHQ question on urgency sensation.

Pelvic floor disorders in women were assessed with 2 separate composite questionnaires: The Pelvic Floor Distress Inventory (PFDI) and the Pelvic Floor Impact Questionnaire (PFIQ). ${ }^{19}$ Each questionnaire had 3 scales (urinary, colorectal, and pelvic organ prolapse), and both have been shown to be psychometrically valid, reliable, and responsive to change. ${ }^{19,22}$ The 46-item PFDI assessed symptom distress in women with pelvic floor disorders and had 3 scales: the Urinary Distress Inventory

TABLE 1. Characteristics of Women with Fecal Incontinence Alone in Comparison to Women with Fecal Incontinence and Irritable Bowel Syndrome

\begin{tabular}{|c|c|c|c|}
\hline Characteristic & $\begin{array}{l}\text { Fecal Incontinence } \\
\text { Alone, } n=82\end{array}$ & $\begin{array}{l}\text { Fecal Incontinence } \\
\text { and Irritable Bowel Syndrome, } \mathbf{n}=\mathbf{3 7}\end{array}$ & $\boldsymbol{P}$ \\
\hline Age, yrs & $59.5 \pm 13.2$ & $54.1 \pm 14.9$ & 0.05 \\
\hline Race & & & 0.92 \\
\hline White/Caucasian & $70(85.4 \%)$ & $31(83.8 \%)$ & \\
\hline Black/African American & $9(11.0 \%)$ & $5(13.5 \%)$ & \\
\hline Asian & $2(2.4 \%)$ & 0 & \\
\hline American Indian/Alaskan & 0 & 0 & \\
\hline Native American & 0 & $1(2.7 \%)$ & \\
\hline Other & $1(1.2 \%)$ & 0 & \\
\hline Ethnicity & & & 0.50 \\
\hline Hispanic & $6(7.3 \%)$ & $4(10.8 \%)$ & \\
\hline Non-Hispanic & $76(92.7 \%)$ & $33(89.2 \%)$ & \\
\hline Body mass index, $\mathrm{kg} / \mathrm{m}^{2}$ & $28.8 \pm 7.3$ & $29.0 \pm 5.7$ & 0.88 \\
\hline Parity & $2.9 \pm 1.4$ & $2.8 \pm 1.6$ & 0.73 \\
\hline Menopausal status & & & 0.04 \\
\hline Premenopausal & $13(15.9 \%)$ & $13(35.1 \%)$ & \\
\hline Postmenopausal & $65(79.3 \%)$ & $24(64.9 \%)$ & \\
\hline Unsure & $4(4.9 \%)$ & 0 & \\
\hline Prior anorectal surgery & $11(13.4 \%)$ & $5(13.5 \%)$ & 0.99 \\
\hline Medication usage for diarrhea & $10(12.2 \%)$ & $7(18.9 \%)$ & 0.33 \\
\hline Medication usage for constipation & $5(6.1 \%)$ & $3(8.1 \%)$ & 0.70 \\
\hline Stool consistency, Bristol Stool Form Scale, Range 1-7 & $\mathrm{n}=80$ & $\mathrm{n}=36$ & 0.82 \\
\hline Categories 1-2 (hard stool) & $16(20.0 \%)$ & $6(16.7 \%)$ & \\
\hline Categories 3-5 (normal stool) & $48(60.0 \%)$ & $21(58.3 \%)$ & \\
\hline Categories 6-7 (loose stool) & $16(20.0 \%)$ & $9(25.0 \%)$ & \\
\hline Strong fecal urgency sensation & $\mathrm{n}=73$ & $\mathrm{n}=32$ & 0.08 \\
\hline Often/always & $22(31.0 \%)$ & $16(50.0 \%)$ & \\
\hline Never/rarely/sometimes & $49(69.0 \%)$ & $16(50.0 \%)$ & \\
\hline
\end{tabular}

All data presented as $\mathrm{n}(\%)$ or mean \pm standard deviation. 
TABLE 2. Relationship Between Participants Being Told They Have IBS by a Medical Professional and Meeting Rome III Clinical Criteria for IBS

\begin{tabular}{lcc}
\hline \multirow{2}{*}{$\begin{array}{l}\text { Medical Professional } \\
\text { Told Participants }\end{array}$} & $\begin{array}{c}\text { Rome III Clinical Criteria for Irritable } \\
\text { Bowel Syndrome }\end{array}$ \\
\cline { 2 - 3 } They Have IBS (N=111) & Yes, $\mathbf{n = 3 5}$ & No, $\mathbf{n = 7 6}$ \\
\hline Yes, $\mathrm{n}=24$ & $12(10.8 \%)$ & $12(10.8 \%)$ \\
No, $\mathrm{n}=87$ & $23(20.7 \%)$ & $64(57.7 \%)$ \\
\hline
\end{tabular}

(UDI; range, 0-300), the Pelvic Organ Prolapse Distress Inventory (range, 0-300), and the Colorectal-Anal Distress Inventory (range, 0-400). ${ }^{19}$ Similarly, the PFIQ measured the impact of bladder, vaginal prolapse, and bowel symptoms on a woman's daily activities, relationships and emotions; it was composed of 3 scales of 31 questions each: the Urinary Impact Questionnaire (range, 0-400), the Pelvic Organ Prolapse Impact Questionnaire (range, 0-400), and the Colorectal-Anal Impact Questionnaire (range, 0-400). ${ }^{19}$ In all measures, a higher score indicated worse symptom bother or greater impact of symptoms on daily functioning or condition-specific QOL.

Participants completed the SF-12, a shortened validated version of the SF-36, to measure the impact on health-related QOL. ${ }^{21}$ The SF-12 had 2 summary scales, the mental and physical components (range, 0-100), where a higher score indicated better QOL.

Demographic and medical history variables were collected at baseline. Demographic variables included age, race, education, and type of medical insurance. Body mass index was obtained from self-reported height and weight values and recorded in kilogram per square meter $\left(\mathrm{kg} / \mathrm{m}^{2}\right)$. Self-reported medical history included parity, diabetes, connective tissue disease, and prior FI treatments, including prior physical therapy, biofeedback, and surgery. The Bristol Stool Form Scale was assessed at baseline to characterize the most common type of stool consistency and categorized as 1 to 2 constipated stool types, 3 to 5 normal stool types, and 6 to 7 loose stool types. ${ }^{23}$
Statistical analysis included a comparison of demographic and medical history variables among women who met IBS clinical criteria versus those who did not have IBS before treatment for FI using 2-sample $t$ tests or Fisher exact tests. Women who had complete questionnaire data at baseline were part of the analytic sample. Questionnaire scores before any treatments were compared using 2-sided $t$ tests. $P \leq 0.05$ or less were considered statistically significant.

\section{RESULTS}

Of the 133 women with monthly FI who consented to participate in the study, 119 women $(89.5 \%)$ had complete data on Rome III IBS questions. Among women with FI who completed IBS questionnaires, 37 (31\%; 95\% confidence interval [CI], $22.9 \%-40.2 \%$ ) met Rome III clinical criteria for IBS with IBS symptoms occurring at least monthly, and 16 (14\%; 95\% CI, $7.9 \%-20.9 \%$ ) had IBS symptoms occurring at least weekly. The most common IBS subtype was IBS-mixed (15/37, 41\%; 95\% CI, $24.8 \%-57.9 \%$ ), followed by IBS-diarrhea $(13 / 37,35 \%$; $95 \%$ CI, $20.2 \%-52.5 \%)$, and IBS-constipation $(8 / 37,22 \%$; $95 \% \mathrm{CI}$, $9.8 \%-38.2 \%)$, with one participant IBS-unclassified (1/37, 3\%; $95 \%$ CI, $0.1 \%-14.2 \%$ ) based on the clinical criteria.

Data in Table 1 showed that women with IBS and FI compared to FI alone had no significant differences $(P>0.05)$ in baseline sociodemographic characteristics. Women with IBS and FI and those with FI alone did not have any differences in prior treatments to manage stool consistency and had similar average stool consistency ratings in the normal range. Menopausal status was different among women with FI and IBS with more women reporting being premenopausal than women with FI alone $(P=0.04)$.

Twenty-four out of 111 patients reported that they had previously been diagnosed with IBS. Among women who met Rome IBS clinical criteria, 23/35 (66\%) had never had a diagnosis of IBS, and half of all women who had had a diagnosis (12/24) did not meet the clinical criteria at the time of this study (Table 2).

Women with IBS and FI had worse mean scores on all questionnaires that measured FI symptom severity, condition-specific QOL, and health-related QOL compared to those with FI alone (Table 3). Specifically, women with IBS and FI compared to FI alone had significantly worse mean total scores for the MMHQ

TABLE 3. Differences in Validated Questionnaires, Mean \pm SD, Among Women with FI Alone Compared to Women with FI and IBS

\begin{tabular}{|c|c|c|c|}
\hline Questionnaires (Score Range) & FI Alone, $n=82$ & FI and IBS, $\mathbf{n}=\mathbf{3 7}$ & $P^{*}$ \\
\hline \multicolumn{4}{|l|}{ Condition-specific Severity } \\
\hline Fecal Incontinence Severity Index (range, 0-61) & $26.3 \pm 11.0 \mathrm{n}=73$ & $28.3 \pm 12.7 n=33$ & 0.41 \\
\hline \multicolumn{4}{|l|}{ Pelvic Floor Disorders Inventory } \\
\hline Urinary Distress Inventory $(0-300)$ & $73.2 \pm 59.9 \mathrm{n}=73$ & $94.7 \pm 71.6 n=33$ & 0.11 \\
\hline Pelvic Organ Prolapse Distress Inventory (0-300) & $71.5 \pm 61.1 \mathrm{n}=73$ & $110.8 \pm 68.9 \mathrm{n}=33$ & 0.004 \\
\hline Colorectal Anal Distress Inventory $(0-400)$ & $124.4 \pm 76.8 \mathrm{n}=71$ & $157.3 \pm 84.4 \mathrm{n}=33$ & 0.05 \\
\hline \multicolumn{4}{|l|}{ Condition-specific Quality of Life } \\
\hline Modified Manchester Health Questionnaire (0-100) & $33.4 \pm 22.7 n=64$ & $47.2 \pm 24.5 \mathrm{n}=29$ & 0.01 \\
\hline \multicolumn{4}{|l|}{ Pelvic Floor Impact Questionnaire (PFIQ) } \\
\hline Urinary Impact Questionnaire $(0-400)$ & $104.9 \pm 109.6 \mathrm{n}=71$ & $144.1 \pm 126.7 \mathrm{n}=31$ & 0.12 \\
\hline Pelvic Organ Prolapse Impact Questionnaire $(0-400)$ & $59.1 \pm 100.1 \mathrm{n}=71$ & $96.9 \pm 122.7 \mathrm{n}=30$ & 0.11 \\
\hline Colorectal Anal Impact Questionnaire (0-400) & $143.9 \pm 112.0 \mathrm{n}=71$ & $199.2 \pm 127.8 \mathrm{n}=33$ & 0.03 \\
\hline \multicolumn{4}{|l|}{ Health-related QOL (higher score represents better QOL) } \\
\hline SF-12 Physical Composite Score $(0-100)$ & $45.5 \pm 10.2 \mathrm{n}=70$ & $42.4 \pm 12.6 n=29$ & 0.20 \\
\hline SF-12 Mental Composite Score $(0-100)$ & $45.1 \pm 12.1 \mathrm{n}=70$ & $41.0 \pm 13.5 n=29$ & 0.14 \\
\hline
\end{tabular}

*Two-sided Student $t$ test used for comparison between groups. Questionnaire scores are presented as mean scores \pm standard deviations. 


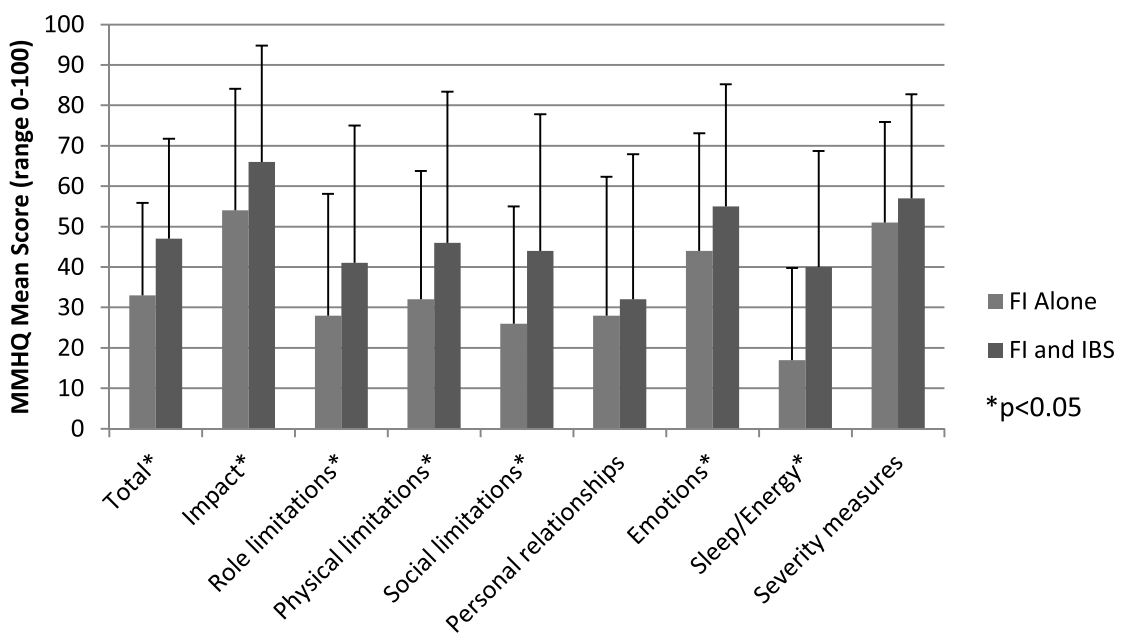

FIGURE 1. Total and Subscale Modified Manchester Health Questionnaire scores among focally incontinent women with and without irritable bowel syndrome. Bars above the columns represent standard deviation of scores.

(47.2 vs $33.4 ; P=0.005)$. Pelvic organ prolapse symptom impact ( 96.9 vs $59.1, P=0.05$ ) and colorectal anal subscales of the PFDI and PFIQ were also significantly worse (199.2 vs 143.9; $P=0.02$ ) among women with IBS and FI compared to FI alone. When evaluating the 8 domain subscales for the MMHQ (Fig. 1), women with IBS and FI were more likely $(P<0.05)$ to endorse having a negative overall impact, more role limitations, physical limitations, social limitations, and a greater negative impact on sleep and emotions.

\section{DISCUSSION}

One third of women presenting to referral-based specialty care clinics with FI had IBS, which was higher than the prevalence of IBS found among women in the general population (3\%-20\%). ${ }^{4}$ According to Rome III criteria, $76 \%$ of the women with IBS and FI met clinical criteria for IBS-mixed and IBSdiarrhea subtypes. Interestingly, $66 \%(23 / 35)$ of women who met Rome IBS clinical criteria had never been told by their health care providers that they had IBS. Women with IBS and FI were more likely to have a greater negative impact on condition-specific QOL and reported more prolapse symptoms than women with FI alone. Better recognition of IBS and IBS subtypes among women with FI is important when evaluating women with FI. Irritable bowel syndrome is often overlooked, judged, and dismissed, especially in younger women with IBS, where FI rates approach the rates seen in long-term care populations. ${ }^{13}$

In review of the literature, many studies indicated that IBS is more common among women with FI and may be an important associated risk factor for FI. ${ }^{24-26}$ Improving stool consistency and bowel movement frequency is an important consideration for women with IBS and FI. In a study among communitydwelling women in Olmstead County, Minnesota, women with bowel disturbances (including diarrhea, presence of IBS, and having a prior cholecystectomy) had a greater odds for developing late-onset FI (after 40 years of age) compared to women who reported obstetrical risk factors (type of delivery, obstetrical injuries). ${ }^{26}$ Others have confirmed that diarrhea and urgency are important factors contributing to FI among women with IBS, along with obstetrical sphincter injuries. ${ }^{26}$ In another study among women presenting for FI treatment to a colorectal surgery clinic, women had a mean age of 57 years, and $44 \%$ met Rome II criteria for IBS. ${ }^{27}$ The authors concluded that conservative nonsurgical treatments may be more appropriate for the women with IBS and FI given that patients with IBS tend to have more severe symptoms. ${ }^{27}$ Given the similar mean age of the women participating in this cohort to other studies, better recognition and treatment of bowel disturbances and IBS may help improve outcomes.

Additionally, understanding the impact of IBS on FI severity and QOL may have important implications for future FI intervention trials. In review of the literature on FI intervention trials, participants with IBS have not been excluded from trials involving dietary modifications with fiber, medications, biofeedback, and sacral nerve stimulation. ${ }^{28-32}$ Recognition of IBS and IBS subtypes that may be more amenable to conservative treatments may be necessary before proceeding with more invasive treatment modalities in women with FI, such as anal injections, anal sphincter surgery, and sacral nerve stimulation. We recommend that future FI intervention studies consider identification of IBS and possibly controlling for the presence of IBS in analytic models.

We found that women with IBS and FI have poorer condition-specific QOL for FI and other colorectal symptoms. However, compared to women with FI alone, women in this cohort with IBS and FI did not report poorer health-related QOL as measured by SF-12 scores. In other studies, women with IBS and FI as individual conditions have reported a significant negative impact on health-related QOL. ${ }^{24,33}$ We speculate that women with IBS and FI may have poorer condition-specific QOL owing to the pain component of IBS, potentially more stool consistency alterations, and the perception that an IBS diagnosis has few treatment options. We also found that women in this cohort with IBS and FI were more likely to report prolapse symptoms, which may also have a negative effect on QOL. In this cohort study, we did not capture physical examination data and cannot comment on the degree of prolapse or the location of prolapse (cystocele or rectocele). Others have found that pelvic floor symptoms, in addition to IBS, are common among women with FI. ${ }^{14,26}$ Possibly, alterations in stool consistency along with pelvic floor weakness can affect the presence of a rectocele. Future studies are needed to evaluate the relationship between stool consistency and having vaginal prolapse.

Our study has several strengths including the prospective multicenter cohort design, which accounts for potential regional variations in access to care, the use of validated questionnaires, 
and the use of the Rome III criteria for IBS diagnosis. In this prospective cohort study, we limited our analysis to bivariate comparisons and did not do multivariable statistical modeling given the small number of women with IBS $(n=37)$. When controlling for other factors, it is possible that the women with IBS and FI may not have significant differences in condition-specific QOL and prolapse symptoms compared to women with FI alone. However, a larger sample of women with IBS and FI would be needed to draw this conclusion. Other limitations involve a participation bias that may exist among women who consented to participate in this study and are seeking treatments for symptoms. In addition, more detailed information on obstetrical factors was not obtained.

In conclusion, our findings suggest that assessment of IBS symptoms, using Rome criteria to diagnose IBS, may be important for women presenting for FI treatment. Clinicians may wish to consider better recognition and treatment of IBS-related symptoms with conservative approaches before progression to more invasive treatments for FI. However, additional evidence is needed to support this approach in clinical care.

\section{REFERENCES}

1. Bharucha AE, Dunivan G, Goode PS, et al. Epidemiology, pathophysiology, and classification of fecal incontinence: state of the science summary for the National Institute of Diabetes and Digestive and Kidney Diseases (NIDDK) Workshop. Am J Gastroenterol 2015;110(1): 127-136.

2. Li FX, Patten SB, Hilsden RJ, et al. Irritable bowel syndrome and health-related quality of life: a population-based study in Calgary, Alberta. Can J Gastroenterol 2003;17(4):259-263.

3. Choung RS, Locke GR 3rd. Epidemiology of IBS. Gastroenterol Clin North Am 2011;40(1):1-10.

4. Sperber AD, Dumitrascu D, Fukudo S, et al. The global prevalence of IBS in adults remains elusive due to the heterogeneity of studies: a Rome Foundation working team literature review. Gut 2016. [Epub ahead of print].

5. Bharucha AE, Zinsmeister AR, Locke GR, et al. Symptoms and quality of life in community women with fecal incontinence. Clin Gastroenterol Hepatol 2006;4(8):1004-1009.

6. Longstreth GF, Thompson WG, Chey WD, et al. Functional bowel disorders. Gastroenterology 2006;130(5):1480-1491.

7. Mayer EA. Clinical practice. Irritable bowel syndrome. N Engl JMed 2008; 358(16):1692-1699.

8. Chey WD, Kurlander J, Eswaran S. Irritable bowel syndrome: a clinical review. JAMA 2015;313(9):949-958.

9. Rome Foundation, Rome III Diagnostic Questionnaires: Available at: http://www.romecriteria.org/questionnaires/. Accessed February 17, 2016.

10. Robinson BL, Matthews CA, Palsson OS, et al. Obstetric sphincter injury interacts with diarrhea and urgency to increase the risk of fecal incontinence in women with irritable bowel syndrome. Female Pelvic Med Reconstr Surg 2013;19(1):40-45.

11. Donnelly VS, O'Herlihy C, Campbell DM, et al. Postpartum fecal incontinence is more common in women with irritable bowel syndrome. Dis Colon Rectum 1998;41(5):586-589.

12. Ng KS, Nassar N, Hamd K, et al. Prevalence of functional bowel disorders and faecal incontinence: an Australian primary care survey. Colorectal Dis 2015;17(2):150-159.

13. Atarodi S, Rafieian S, Whorwell PJ. Faecal incontinence-the hidden scourge of irritable bowel syndrome: a cross-sectional study. BMJ Open Gastroenterol 2015;1(1):e000002.

14. Jelovsek JE, Barber MD, Paraiso MF, et al. Functional bowel and anorectal disorders in patients with pelvic organ prolapse and incontinence. Am J Obstet Gynecol 2005;193(6):2105-2111.
15. Menees SB, Smith TM, Xu X, et al. Factors associated with symptom severity in women presenting with fecal incontinence. Dis Colon Rectum 2013;56(1):97-102 10.1097/DCR.0b013e31826f8773.

16. Walter S, Hjortswang H, Holmgren K, et al. Association between bowel symptoms, symptom severity, and quality of life in Swedish patients with fecal incontinence. Scand J Gastroenterol 2011;46(1):6-12.

17. Markland A, Wang L, Jelovsek JE, et al. Symptom improvement in women after fecal incontinence treatments: a multicenter cohort study of the pelvic floor disorders network. Female Pelvic Med Reconstr Surg 2015;21:46-52.

18. Rockwood TH, Church JM, Fleshman JW, et al. Patient and surgeon ranking of the severity of symptoms associated with fecal incontinence: the fecal incontinence severity index. Dis Colon Rectum 1999;42(12): $1525-1532$.

19. Barber MD, Kuchibhatla MN, Pieper CF, et al. Psychometric evaluation of 2 comprehensive condition-specific quality of life instruments for women with pelvic floor disorders. Am J Obstet Gynecol 2001;185(6):1388-1395.

20. Kwon S, Visco AG, Fitzgerald MP, et al. Validity and reliability of the Modified Manchester Health Questionnaire in assessing patients with fecal incontinence. Dis Colon Rectum 2005;48(2):323-331.

21. Ware J Jr, Kosinski M, Keller SD. A 12-Item Short-Form Health Survey: construction of scales and preliminary tests of reliability and validity. Med Care 1996;34(3):220-233.

22. Barber MD, Chen Z, Lukacz E, et al. Further validation of the short form versions of the Pelvic Floor Distress Inventory (PFDI) and Pelvic Floor Impact Questionnaire (PFIQ). Neurourol Urodyn 2011;30(4):541-546.

23. Probert CJ, Emmett PM, Heaton KW. Intestinal transit time in the population calculated from self made observations of defecation. J Epidemiol Community Health 1993;47(4):331-333.

24. Boreham MK, Richter HE, Kenton KS, et al. Anal incontinence in women presenting for gynecologic care: prevalence, risk factors, and impact upon quality of life. Am J Obstet Gynecol 2005;192(5):1637-1642.

25. Varma MG, Brown JS, Creasman JM, et al. Fecal incontinence in females older than aged 40 years: who is at risk? Dis Colon Rectum 2006;49(6): $841-851$.

26. Bharucha AE, Zinsmeister AR, Schleck CD, et al. Bowel disturbances are the most important risk factors for late onset fecal incontinence: a population-based case-control study in women. Gastroenterology 2010; 139(5):1559-1566.

27. Chaudhary BN, Chadwick M, Roe AM. Selecting patients with faecal incontinence for anal sphincter surgery: the influence of irritable bowel syndrome. Colorectal Dis 2010;12(8):750-3.

28. Bliss DZ, Savik K, Jung HJ, et al. Dietary fiber supplementation for fecal incontinence: a randomized clinical trial. Res Nurs Health 2014;37(5): 367-378.

29. Markland AD, Burgio KL, Whitehead WE, et al. Loperamide versus psyllium fiber for treatment of fecal incontinence: the Fecal Incontinence Prescription (Rx) Management (FIRM) Randomized Clinical Trial. Dis Colon Rectum 2015;58(10):983-993.

30. Heymen S, Scarlett Y, Jones K, et al. Randomized controlled trial shows biofeedback to be superior to pelvic floor exercises for fecal incontinence. Dis Colon Rectum 2009;52(10):1730-1737.

31. Tjandra JJ, Chan MK, Yeh CH, et al. Sacral nerve stimulation is more effective than optimal medical therapy for severe fecal incontinence: a randomized, controlled study. Dis Colon Rectum 2008;51(5):494-502.

32. Bols E, Berghmans B, de Bie R, et al. Rectal balloon training as add-on therapy to pelvic floor muscle training in adults with fecal incontinence: a randomized controlled trial. Neurourol Urodyn 2012;31(1):132-138.

33. Dean BB, Aguilar D, Barghout V, et al. Impairment in work productivity and health-related quality of life in patients with IBS. Am J Manag Care 2005;11(Suppl 1):S17-S26. 